\title{
Determination of allelopathic potentials in plant species in Sino-Japanese floristic region by sandwich method and dish pack method
}

\author{
Kwame Sarpong Appiah ${ }^{1}$, Zhenhao Li ${ }^{1}$, Ren-Sen Zeng ${ }^{2,3}$, Shiming Luo ${ }^{2}$, Yosei Oikawa ${ }^{1}$, Yoshiharu Fujii ${ }^{1}$ * \\ ${ }^{1}$ Department of International Environmental And Agricultural Science, Tokyo University of Agriculture and Technology, Japan \\ ${ }^{2}$ Institute of Tropical and Subtropical Ecology, South China Agricultural University, China \\ ${ }^{3}$ College of Crop Science, Fujian Agriculture and Forestry University, China \\ *Corresponding authorE-mail: yfujii@cc.tuat.ac.jp
}

Copyright () 2015 Kwame Sarpong Appiah et al. This is an open access article distributed under the Creative Commons Attribution License, which permits unrestricted use, distribution, and reproduction in any medium, provided the original work is properly cited.

\begin{abstract}
The Sino-Japanese Floristic Region appears as one of the major centers of development of higher plants. This region have been relevant for the study of evolution and systematics of many flowering plants. The taxonomic richness of endemic plant species in this region have survived several years of extreme climate conditions. Endemic mountainous plant species that have survived extreme climate conditions are of allelopathic and medicinal interest. For this reason, 251 plant species collected from the Sino-Japanese Floristic Region were screened for allelopathic plant species. Sandwich method and dish pack method were respectively used to screen plant leaf leachates and volatile materials with lettuce (Lactuca sativa CV. Great Lakes 366) as receptor plant. Among the 84 species that showed inhibitory effect on lettuce radicle elongation in our sandwich bioassay, Photinia glabra showed complete inhibition of lettuce radicle elongation ( $0 \%$ radicle elongation). In the dish pack bioassay, Photinia glabra, Liquidambar styraciflua, and Cinnamomum camphora $(90.6 \%, 61.4 \%$, and 50.2\% respectively) were among the nine species that were observed with strong inhibitory effect on lettuce radicle growth. On the other hand, nine other species promoted lettuce radicle growth when compared to the control. Aesculus turbinata and Quercus gilva were the species with the highest growth stimulatory effect (33.0\% and $16.1 \%$ respectively). We hereby present Photinia glabra as an allelopathic candidate species for both leachate and volatile compounds.
\end{abstract}

Keywords: Allelochemicals; Dish Pack Method; Elongation; Leaf Leachates; Sandwich Method; Sino-Japanese Floristic Region.

\section{Introduction}

Some living organisms especially plants, have the inherent ability to interfere with biological activities of other organism(s) in their immediate vicinity by releasing certain compounds, this phenomenon is termed as allelopathy. The term allelopathy describes beneficial and mostly harmful natural interactions between organisms due to the release of bioactive secondary metabolites from the donor organism. These secondary metabolites associated with this phenomenon are called allelochemicals which are mostly introduced into the environment through volatilization, leaching, root exudation, and/or by the decomposition of plant residues [1]. Majority of allelochemicals are products of secondary metabolism with a few resulting from primary metabolism [2]. From an ecological perspective, allelopathy may play an important role in the process of biological invasion. Some invasive plant species are perceived to be successful because they possess novel compounds that function as allelopathic agents or as mediators of the new plantplant interactions [3]. Some effects of allelochemicals on the growth and development of susceptible plants include; reduced radicle and shoot extension, darkened and/or swollen seeds, curling of root axis, discoloration of seeds, lack of root hairs, necrosis, increased number of seminal roots, and reduced dry weight accumulation among others [4]. Modern agricultural practices have succeeded due to the discovery and adoption of agrochemicals for pest control. However, there have been 452 unique cases of herbicide resistant/tolerant weeds among 245 species [5]. Nonetheless, it is difficult 
to estimate the cost associated with yield losses due to only herbicide-resistant/tolerant weeds [6]. Due to the increasing number of herbicide-resistant/tolerant weeds and environmental concerns about the inappropriate use of synthetic herbicides, efforts have been made towards developing alternate sustainable weed management strategies. Plants that are able to suppress and/or eliminate competing plant species have received much attention, and the possibilities of using compounds from such plants as selective natural herbicides have increased [7,8]. Isolated bioactive substances (allelochemicals) from plants are therefore important sources for alternate agrochemicals which could help reduce some of the problems arising from poor cultural practices and excessive use of synthetic pesticides [9]. These natural agrochemicals, compared to their synthetic counterparts are expected to have shorter half-lives in the environment and hence considered to be more environmentally friendly [10]. Over the last decade, there have been a growing market for products from organic farming [11]. Consequently, current researches in weed management have focused much attention on the use of natural products (allelochemicals) as natural pesticides in order to reduce the effects of synthetic pesticides on environment and human health, and to promote sustainable agriculture [12]. These have called for the screening for growth inhibitory plants and the subsequent isolation of their active compounds. This study focused on plants in the Sino-Japanese Floristic Region in East Asia which have one of the most diverse temperate floras in the world. The flora of this region holds special interest for the study of the history of temperate floras of the northern hemisphere. Several plant species of different genera have been reported to be endemic in this region [13]. Qian, [14] reported that the taxonomic richness of seed plants of East Asia is significantly more diverse compared to North America with approximately twice as many plant species as eastern North America, which holds similar size and environment. High physiographical heterogeneity is considered to be of major influence on the extremely high floral diversity within the Sino-Japanese Floristic Region [15]. During the exceptionally cold periods of climate change, the series of mountains (usually with elevations of about $2000 \mathrm{~m}$ ) in this region provided diverse habitats allowing for species survival. Cool environments at higher elevations are suitable for survival of relict populations in modern subtropics. These relict population may however had allowed for the divergence between extant populations [16]. Recently, the allelopathic potential of certain plant species especially those with medicinal properties have been reported. In this study, we present the comprehensive screening of allelopathic activity of some plants in this region using the sandwich and dish pack methods. The basis of current weed control researches towards identifying potent bioactive compound(s) for weed control is the screening of large quantities of plants. Potential allelopathic candidate species would be identified from the screening process to pave way for further researches. We examined 256 plant samples from 251 different plant species for their allelopathic potentials under laboratory conditions. This report only focused on identifying and introducing allelopathic potentials in some plant species of Sino-Japanese region, while another report will focus on the identification of allelopathic compounds in species that exhibited strong allelopathic potentials for growth inhibitors.

\section{Materials and methods}

\subsection{Plant samples and preparation}

The collection of plant samples focused on a part of Japan and China called the Sino-Japanese Floristic Region. A total of 256 plant samples were collected from seven different locations; including the campus of Tokyo University of Agriculture and Technology (TUAT), Tsukuba Botanical Gardens (TKBG), Tokyo Medicinal Botanical Garden (TMBG), Wuhan Botanical Garden (WHBG), Kunming Botanical Garden (KMBG), South China Botanical Garden, (SCBG), and South China University of Agriculture (SCUA). The leaves and other parts of each plant species were freshly collected, placed in separate paper bags and oven-dried $\left(60^{\circ} \mathrm{C}\right.$ for 24 hours). The samples were then kept in an air-tight box until further use. The oven-dried samples were used for laboratory studies in the Laboratory of International Agro-Biological Resources and Allelopathy at Tokyo University of Agriculture and Technology, Japan.

\subsection{Sandwich method}

The sandwich method adopted from Fujii et al., [17] was used to determine the allelopathic activity of leachates from selected donor plant leaves. This method have been used [18, 19, 20] to screen large quantity of plants and is effective in determining allelopathic activities by plant leachates under laboratory conditions. Using this method, 251 plant samples (245 species) were screened. Using multi-well plastic dish, the sandwich method was set up as shown in Fig. 1. Treatments were replicated three times and data presented as the mean of the three replicates. Agar with no plant material was set as the untreated control. The multi-well plastic plates were completely randomized in an incubator (NTS Model MI-25S) at $25^{\circ} \mathrm{C}$ for three days after which radicle and hypocotyl lengths were measured.

\subsection{Dish pack method}

Fujii et al., [21] adopted this approach to screen for the presence of volatile allelochemicals from plant species. This method is widely used [22] because it determines the presence of volatile allelochemicals in plants very quickly. Using 
this method, 69 plant species were screened for possible volatile substances that can influence (promote or inhibit) the growth of lettuce. Multi-well plastic dishes with 6 wells (36 mm×18 mm each) were used in this experiment. The distances from the center of the source well (where plant sample was placed) to the center of other wells were 41, 58, 82, and $92 \mathrm{~mm}$ (Fig. 2). The source well was filled with $200 \mathrm{mg}$ of oven-dried plant material, while filter papers were laid in the other wells and $0.75 \mathrm{ml}$ of distilled water was added to each of the wells containing filter paper. The control treatment did not contain any plant sample at the source well. Seven lettuce seeds (Lactuca sativa var. Great Lakes 366) were placed on the filter paper in each well. The multi-well dishes were tightly sealed using cellophane tape to avoid desiccation and loss of volatile compounds. To exclude light, aluminum foils were wrapped around the dishes and placed in an incubator (NTS Model MI-25S) at $25^{\circ} \mathrm{C}$ for three days. The radicle and hypocotyl lengths were measured and recorded after 3 days of incubation and compared to that of the control. The degree of inhibition were estimated by the relationship between lettuce seedling growth inhibition and its distance from the source well.

A

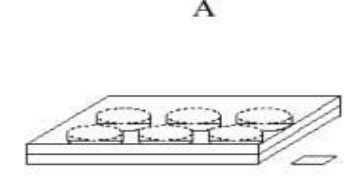

B

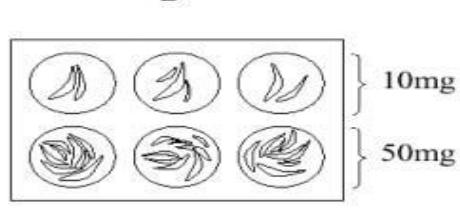

E

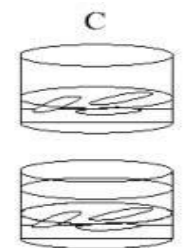

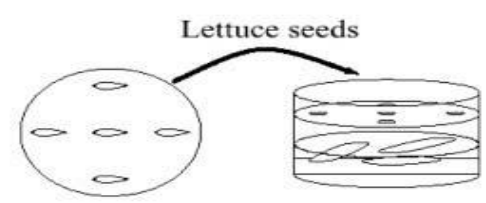

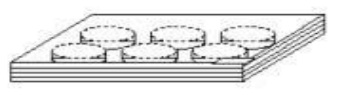

Fig. 1: Sandwich Method: (A) Multi-well plastic plate with six wells; (B) 10 Or $50 \mathrm{mg}$ dried plant material placed in each well of the multi-well plastic plate; (C) Addition of $5 \mathrm{~mL}$ plus $5 \mathrm{~mL}$ agar (Nacalai Tesque Agar Powder, $0.75 \%$ w/v autoclaved for $20 \mathrm{minutes}$ at $120^{\circ} \mathrm{C}$ ) in two layers on the oven-dried plant material; (D) Five seeds (Lactuca Sativa Var. Great Lakes 366) Lettuce seeds vertically placed; (E) Covered with plastic tape and appropriately labelled the multi-well plastic plates for incubation in dark conditions [17].

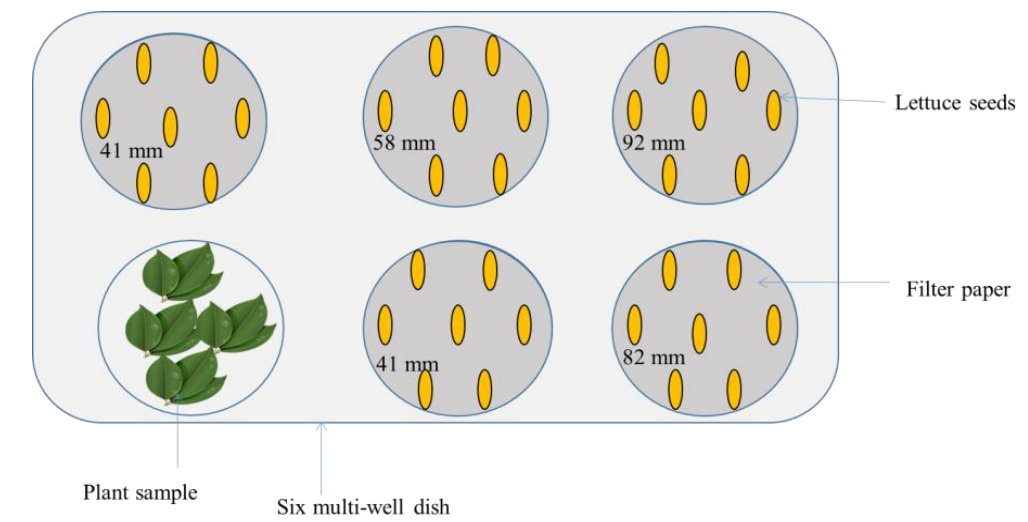

Fig. 2: View from top of Multi-well plastic plate used to test for plant allelopathy through volatile substances.

\subsection{Statistical analysis}

The experimental set-up was arranged in a complete randomized design with three replicates. In the statistical analysis, evaluation of the means, standard deviation (SD), and SD variance (SDV) were done using Microsoft Excel 2007.

Elongation $\%=\underline{(\text { Average length of treatment radicle/hypocotyl })}$

(Average length of control radicle/hypocotyl)

Inhibitory $\%=100-($ Average length of treatment radicle/hypocotyl $)$ (Average length of control radicle/hypocotyl)

\section{Results}

\subsection{Allelopathic effects of leachates from oven-dried plant materials on lettuce}

The percentage elongation of radicle and hypocotyl of lettuce seedlings (1) as affected by leachates from 245 plant species based on sandwich method is shown in Table 1. In this study, the radicle elongations percentages of lettuce 
seedlings were in the range $0-123 \%$ and $0-105 \%$ of the untreated control when respectively treated with $10 \mathrm{mg}$ and 50 $\mathrm{mg}$ of oven-dried leaves. In both $10 \mathrm{mg}$ and $50 \mathrm{mg}$ of oven-dried leaf treatment, lettuce radicle elongations were inhibited more than hypocotyl elongations. With respect to $10 \mathrm{mg}$ oven dried leaves treatment, it was observed that 84 species caused significant inhibition on lettuce radicle as evaluated using standard deviation variance (SDV). The families with the highest number of different plant species examined were Magnoliaceae (16 species), Rosaceae, and Fabaceae (11 species each), Fagaceae (8 species) with Oleaceae, Moraceae, and Araliaceae have 7 species each. Only the Rosaceae and Amaryllidaceae families had two species that had lettuce radicle elongation less than $29 \%$ of control with Anacardiaceae and Malvaceae having one species each. Further, Boraginaceae, Alistolochiaceae, Euphorbiaceae, Berberidiaceae, Taxaceae, Magnoliaceae, Hemerocallidaceae, and Rutaceae (one species each) showed lettuce radicle elongation in the range $29.5-39.8 \%$ of control. It was also found that the oven-dried leaves of six species showed the strongest inhibitory activity on lettuce seedling, showing radicle elongation in the range of $0-29 \%$ of the untreated control for $10 \mathrm{mg}$ treatment. These species include; Photinia glabra, Dracontomelon duperreanum, Hibiscus syriacus, Amygdalus persica, Lycoris aurea, and Lycoris radiata. Eight other species (Cordia dichotoma, Asarum nipponicum, Bischofia polycarpa, Mahonia lomariifolia, Taxus wallichiana, Magnolia liliiflora, Hemerocallis fulva, and Acronychia pedunculata) showed strong inhibitory activity on lettuce seedling with radicle elongation in the range of $29.5-39.8 \%$ of the untreated control for $10 \mathrm{mg}$ treatment. In lettuce radicle elongation of $38.9-50.2 \%$ of the untreated control, 18 different species were observed when treated with $10 \mathrm{mg}$ oven-dried leaves. The lowest inhibitory activity in this study was observed in 52 plant species with lettuce radicle elongation for $10 \mathrm{mg}$ treatment in the range of 50.3-60.6\% of the untreated control. In terms of inhibition on lettuce hypocotyl elongation, only two species P. glabra and A. persica (both Rosaceae) could cause the strongest reduction $(<29.5 \%)$ in this study. Among the 251 plant samples evaluated, only P. glabra could completely reduce both lettuce radicle and hypocotyl elongations to $0 \%$ for both $10 \mathrm{mg}$ and $50 \mathrm{mg}$ oven-dried leaves treatment. Photinia glabra (Rosaceae) was ranked the strongest inhibitory plant species among the evaluated species using the sandwich method.

\subsection{Effects of volatiles compounds from plant species on lettuce seedlings in dish pack method}

Table 2 shows the effects (inhibition or promotion) on radicle and hypocotyl of lettuce seedlings that were grown in dish packs containing oven-dried leaves from 69 different plant species. The effects of the plant leaves on growth of lettuce radicle and hypocotyl (2) were presented either as promotion or inhibition. Lettuce radicle growth values indicated negative represent promotional effect when compared to the corresponding control. Our results indicate that among the 69 plant species tested, lettuce radicle growth was either inhibited or stimulated by 9 different species each when compared to the control. Strongest inhibitory effects were shown in seven families, including Rosaceae (two), Taxadiaceae (two), with Altingiaceae, Lauraceae, Pinaceae, Rubiaceae, and Juglandaceae having one species each for different plant species. Only Photinia glabra was observed among the 69 plant species tested to have inhibited lettuce radicle growth more than $90 \%$. It was also observed that two other species (Liquidambar styraciflua and Cinnamomum camphora) showed lettuce radicle growth inhibition in the range of 50-62\%. Six other species including, Metasequoia glyptostroboides, Sciadopitys verticillata, Amygdalus persica, Pinus parviflora, Platycarya strobilacea, and Gardenia sootepensis demonstrated lettuce radicle inhibitory effect in the region of 31-39\%. Moreover, Aesculus turbinata showed stimulatory effect on lettuce growth more than 25\%, whereas Quercus gilva, Diospyros kaki, Prunus buergeriana, Cephalotaxus fortunei, and Fraxinus longicuspis demonstrated lettuce growth stimulation in the range of 10-24\%. Polyalthia longifolia, Magnolia obovata, and Acer mono, showed the least stimulatory effect (6.0-9.8\%) on lettuce radicle growth.

\section{Discussion}

Our study indicated that among 251 plant species studied, 10 species showed very strong inhibitory activity on radicle and hypocotyl lengths of lettuce seedling. Currently, there have been no allelopathic reports on six of these species (Photinia glabra, Liquidambar styraciflua, Hibiscus syriacus, Lycoris aurea, Cordia dichotoma, and Asarum nipponicum). Nonetheless, these plants contain some phytochemicals that are linked to phytotoxicity and the inhibition effects observed in these plant species may be due to these compounds or some unknown chemical constituents. We however introduce these compounds in this report. Another report will focus on the identification of bioactive compounds with allelopathic capabilities associated with some of these plant species. Among the species of the Rosaceae family in this study, Photinia glabra had the greatest inhibition on lettuce radicle growth in both sandwich and dish pack methods. P. glabra is native to Japan and have been widely planted for its attractive bright-red new leaf growth and grows 15 to 20 feet in height [23]. The leaves of $P$. glabra produced two biphenyl compounds when inoculated with fungal spores and treated with $\mathrm{HgCl}_{2}$. These two biphenyl compounds (2'-methoxyaucuparin and 4'methoxyaucuparin) are reasoned to be produced in response to microbial attack [24]. These phytoalexins from $P$. glabra and other plant species from the Rosaceae family can inhibit several pathogens especially fungi but their usefulness are however still limited [25]. Hirai et al., [26] reported that plants in the Rosaceae family contain sorbitol which is synthesized from glucose-6-phosphate during photosynthesis in the leaves of these plants. Ishikura, [27] reported that the fruits of $P$. glabra contain anthocyanin identified as cyaniding 3-monoglucoside. 
Table 1: Radicle and hypocotyl elongation percentages of lettuce seedlings grown on agar gel containing oven-dried plant materials tested using the sandwich method.

\begin{tabular}{|c|c|c|c|c|c|c|c|}
\hline \multirow{3}{*}{ Plant families } & \multirow{3}{*}{$\mathrm{POC}$} & \multirow{3}{*}{ Scientific Name } & \multicolumn{4}{|c|}{ Dry leaf content $\left(10 \mathrm{ml}\right.$ agar $\left.^{-1}\right)$} & \multirow{3}{*}{ Criteria } \\
\hline & & & \multicolumn{2}{|c|}{$10 \mathrm{mg}$} & \multicolumn{2}{|c|}{$50 \mathrm{mg}$} & \\
\hline & & & $\mathrm{R} \%$ & $\mathrm{H} \%$ & $\mathrm{R} \%$ & $\mathrm{H} \%$ & \\
\hline \multirow[t]{2}{*}{ Acanthaceae } & WHUN & Adhatoda vasica Nees & 55.6 & 115 & 24.2 & 91.2 & $*$ \\
\hline & WHUN & Gendarussa vulgaris Nees & 62.2 & 145 & 22.9 & 80.0 & \\
\hline \multirow[t]{5}{*}{ Aceraceae } & TUAT & Acer pictum Thunb. & 55.2 & 92.6 & 26.1 & 83.3 & $*$ \\
\hline & TUAT & Acer buergerianum Miq. & 59.5 & 105 & 22.2 & 70.8 & $*$ \\
\hline & TUAT & Acer cissifolium K. Koch & 61.5 & 88.2 & 16.2 & 43.2 & \\
\hline & TUAT & Acer palmatum Thunb. & 67.4 & 87.5 & 27.2 & 85.8 & \\
\hline & TUAT & Acer diabolicum Blume ex K. Koch & 83.9 & 120 & 26.8 & 81.1 & \\
\hline Acoraceae & WHUN & Acorus gramineus Aiton & 59.3 & 126 & 20.1 & 67.7 & $*$ \\
\hline \multirow[t]{2}{*}{ Actinidiaceae } & TSUK & Actinidia arguta Franch. \& Sav. & 65.6 & 83.8 & 46.7 & 123 & \\
\hline & TSUK & Actinidia rufa Franch. \& Sav. & 88.1 & 111 & 35.0 & 87.1 & \\
\hline Aristolochiaceae & TSUK & Asarum nipponicum F. Maek. & 33.6 & 82.9 & 16.7 & 76.3 & $* * *$ \\
\hline Altingiaceae & TUAT & Liquidambar styraciflua L. & 83.5 & 114 & 59.1 & 95.8 & \\
\hline \multirow[t]{2}{*}{ Amaryllidaceae } & WHUN & Lycoris radiata Herb. & 26.3 & 92.0 & 8.70 & 35.2 & $* * * *$ \\
\hline & SCBG & Lycoris aurea Herb. & 23.2 & 72.5 & 0.0 & 0.0 & $* * * *$ \\
\hline \multirow[t]{2}{*}{ Anacardiaceae } & WHUN & Spondias lakonensis Pierre & 72.8 & 101 & 50.0 & 73.0 & \\
\hline & SCBG & Dracontomelon duperreanum Pierre & 13.1 & 40.4 & 12.6 & 44.8 & $* * * * *$ \\
\hline \multirow[t]{2}{*}{ Annonaceae } & SCBG & Artabotrys hexapetalus (L. f.) Bhandari & 58.1 & 84.2 & 28.3 & 69.0 & $*$ \\
\hline & SCAU & Polyalthia longifolia (Sonn.) Thwaites & 76.0 & 123 & 31.1 & 118 & \\
\hline Apiaceae & KUMN & Peucedanum decumbens Maxim. & 64.4 & 91.4 & 41.6 & 78.5 & \\
\hline \multirow[t]{3}{*}{ Apocynaceae } & SCBG & Alstonia scholaris (L.) R. Br. & 52.1 & 91.9 & 29.9 & 87.6 & $*$ \\
\hline & $\mathrm{SCBG}$ & $\begin{array}{l}\text { Tabernaemontana divaricata (L.) R. Br. } \\
\text { ex Roem. \& Schult. }\end{array}$ & 58.1 & 91.2 & 31.3 & 65.5 & $*$ \\
\hline & $\mathrm{SCBG}$ & Wrightia pubescens $\mathrm{R} . \mathrm{Br}$. & 73.2 & 109 & 49.5 & 97.1 & \\
\hline \multirow[t]{4}{*}{ Aquifoliaceae } & SCBG & Ilex ferruginea Hand.-Mazz. & 56.2 & 110 & 35.5 & 84.9 & $*$ \\
\hline & TUAT & Ilex crenata Thunb. & 76.3 & 127 & 75.4 & 124 & \\
\hline & SCBG & Ilex rotunda Thunb. & 96.3 & 175 & 51.2 & 111 & \\
\hline & TUAT & Ilex integra Thunb. & 98.6 & 120 & 78.2 & 122 & \\
\hline Arecaceae & SCBG & $\begin{array}{l}\text { Livistona fengkaiensis X. W. Wei \& M. } \\
\text { Y. Xiao }\end{array}$ & 56.0 & 97.5 & 42.3 & 80.4 & $*$ \\
\hline \multirow[t]{2}{*}{ Araceae } & SCBG & Alocasia macrorrhizos (L.) G. Don & 60.6 & 122 & 52.0 & 115 & $*$ \\
\hline & WHUN & Pothos chinensis (Raf.) Merr. & 47.6 & 107 & 19.5 & 51.3 & $* *$ \\
\hline \multirow[t]{7}{*}{ Araliaceae } & WHUN & Acanthopanax sessiliflorus Seem. & 46.4 & 116 & 24.4 & 75.4 & $* *$ \\
\hline & SCAU & Schefflera octophylla Harms & 65.9 & 130 & 21.9 & 59.6 & \\
\hline & TUAT & Hedera rhombea Siebold \& Zucc. & 67.2 & 109 & 36.3 & 86.8 & \\
\hline & TSUK & Aralia cordata Thunb. & 107 & 164 & 48.0 & 151 & \\
\hline & KUMN & Acanthopanax simonii C. K. Schneid & 73.2 & 91.4 & 59.8 & 81.5 & \\
\hline & TSUK & Aralia elata (Miq.) Seem. & 86.5 & 109 & 27.1 & 86.5 & \\
\hline & WHUN & Hedera nepalensis K. Koch & 60.4 & 106 & 29.4 & 105 & $*$ \\
\hline Arecaceae & $\mathrm{SCBG}$ & Rhapis excelsa (Thunb.) A. Henry & 61.1 & 94.7 & 30.8 & 72.4 & \\
\hline & SCBG & Arenga tremula Becc. & 82.9 & 109 & 64.1 & 111 & \\
\hline & SCBG & Caryota urens L. & 95.3 & 129 & 91.7 & 149 & \\
\hline & SCAU & Areca triandra Roxb. ex Buch.-Ham. & 114 & 123 & 94.0 & 125 & \\
\hline & $\mathrm{SCBG}$ & Arenga pinnata Merr. & 71.2 & 94.7 & 43.9 & 75.9 & \\
\hline Asparagaceae & TSUK & Hosta sieboldiana (Hook.) Engl. & 43.3 & 59.2 & 34.4 & 58.8 & $* *$ \\
\hline & WHUN & Asparagus albus L. & 105.3 & 134 & 89.0 & 135 & \\
\hline Asteraceae & TSUK & Ligularia fischeri Turcz. & 50.2 & 88.3 & 26.7 & 64.9 & $*$ \\
\hline & TSUK & $\begin{array}{l}\text { Chrysanthemum japonicum (Maxim.) } \\
\text { Makino }\end{array}$ & 71.8 & 106 & 24.0 & 51.5 & \\
\hline & TSUK & Aster ageratoides Turcz. & 85.3 & 110 & 65.2 & 103 & \\
\hline & TSUK & Chrysanthemum pacificum Nakai & 50.9 & 102 & 73.3 & 104 & $*$ \\
\hline & TSUK & Stevia rebaudiana Bertoni. & 111 & 113 & 53.3 & 113 & \\
\hline Berberidaceae & KUMN & Mahonia lomariifolia Takeda & 35.1 & 67.2 & 26.8 & 53.8 & $* * *$ \\
\hline & TSUK & Nandina domestica Thunb. & 47.3 & 68.9 & 26.2 & 47.4 & $* *$ \\
\hline & SCBG & Mahonia fortunei hort. ex Dippel & 54.8 & 76.6 & 29.9 & 81.4 & $*$ \\
\hline Betulaceae & TUAT & Betula platyphylla Sukaczev & 83.7 & 104 & 65.2 & 108 & \\
\hline Bignoniaceae & $\mathrm{SCBG}$ & Mayodendron igneum Kurz & 56.0 & 90.3 & 28.0 & 82.5 & $*$ \\
\hline
\end{tabular}




\begin{tabular}{|c|c|c|c|c|c|c|c|}
\hline \multirow{3}{*}{ Plant families } & \multirow{3}{*}{ POC } & \multirow{3}{*}{ Scientific Name } & \multicolumn{4}{|c|}{ Dry leaf content $\left(10 \mathrm{ml} \mathrm{agar}^{-1}\right)$} & \multirow{3}{*}{ Criteria } \\
\hline & & & \multicolumn{2}{|c|}{$10 \mathrm{mg}$} & \multicolumn{2}{|c|}{$50 \mathrm{mg}$} & \\
\hline & & & $\mathrm{R} \%$ & $\mathrm{H} \%$ & $\mathrm{R} \%$ & $\mathrm{H} \%$ & \\
\hline \multirow[t]{2}{*}{ Bignoniaceae } & WHUN & Tecomaria capensis (Thunb.) Spach & 62.8 & 112 & 38.0 & 120 & \\
\hline & SCBG & $\begin{array}{l}\text { Dolichandrone cauda-felina Benth. \& } \\
\text { Hook. f. }\end{array}$ & 82.4 & 106 & 35.2 & 87.6 & \\
\hline \multirow[t]{2}{*}{ Bombacaceae } & SCBG & Bombax malabaricum DC. & 59.1 & 114 & 49.0 & 79.3 & $*$ \\
\hline & WHUN & $\begin{array}{l}\text { Ceiba speciosa (A. St.-Hil., A. Juss. \& } \\
\text { Cambess.) Ravenna }\end{array}$ & 68.0 & 107 & 26.8 & 90.8 & \\
\hline \multirow[t]{2}{*}{ Boraginaceae } & SCBG & Cordia dichotoma $\mathrm{G}$. Forst. & 30.7 & 75.7 & 17.8 & 73.5 & $* * *$ \\
\hline & SCAU & Ehretia thyrsiflora Nakai & 58.1 & 109 & 40.4 & 96.5 & $*$ \\
\hline Brassicaceae & SCBG & Isatis indigotica Fortune & 53.5 & 123 & 32.8 & 74.1 & $*$ \\
\hline Buxaceae & WHUN & $\begin{array}{l}\text { Buxus sinica (Rehder \& E. H. Wilson) } \\
\text { M. Cheng }\end{array}$ & 55.2 & 81.0 & 31.6 & 75.4 & $*$ \\
\hline Caprifoliaceae & TUAT & Viburnum odoratissimum Ker Gawl. & 78.1 & 99.2 & 65.5 & 124 & \\
\hline \multirow[t]{4}{*}{ Celastraceae } & SCBG & Euonymus bungeanus Maxim. & 45.9 & 84.2 & 47.5 & 129 & $* *$ \\
\hline & WHUN & Perrottetia racemosa (Oliv.) Loes. & 65.1 & 129 & 39.1 & 99.4 & \\
\hline & TUAT & Microtropis japonica Hallier f. & 83.8 & 115 & 74.6 & 116 & \\
\hline & TSUK & Euonymus japonicus L. f. & 95.2 & 117 & 51.2 & 96.8 & \\
\hline Cephalotaxaceae & WHUN & Cephalotaxus fortunei Hook. & 87.9 & 138 & 68.6 & 124 & \\
\hline Cercidiphyllaceae & TUAT & $\begin{array}{l}\text { Cercidiphyllum japonicum Siebold \& } \\
\text { Zucc. }\end{array}$ & 67.3 & 109 & 47.0 & 102 & \\
\hline Chloranthaceae & WHUN & $\begin{array}{l}\text { Sarcandra hainanensis (C. Pei) Swamy } \\
\text { \& I. W. Bailey }\end{array}$ & 76.5 & 142 & 35.6 & 108 & \\
\hline Clusiaceae & TSUK & Hypericum ascyron $\mathrm{L}$. & 94.7 & 92.2 & 47.1 & 87.6 & \\
\hline Convallariaceae & KUMN & Aspidistra elatior Blume & 62.9 & 90.0 & 42.6 & 79.4 & \\
\hline Coriariaceae & TSUK & Coriaria japonica A. Gray & 79.7 & 104 & 46.3 & 90.7 & \\
\hline Cornaceae & TUAT & $\begin{array}{l}\text { Benthamidia japonica (Siebold \& } \\
\text { Zucc.) H. Hara }\end{array}$ & 91.3 & 99.0 & 44.9 & 110 & \\
\hline Corylaceae & TUAT & Carpinus tschonoskii Maxim. & 81.3 & 118 & 78.0 & 121 & \\
\hline \multirow[t]{4}{*}{ Cupressaceae } & TUAT & Juniperus chinensis L. & 88.7 & 102 & 75.1 & 105 & \\
\hline & TSUK & Sequoia sempervirens (D. Don) Endl. & 90.0 & 114 & 57.0 & 119 & \\
\hline & KUMN & $\begin{array}{l}\text { Sabina pingii (W. C. Cheng ex Ferre) } \\
\text { W. C. Cheng \& W. T. Wang }\end{array}$ & 100 & 121 & 91.4 & 117 & \\
\hline & TUAT & $\begin{array}{l}\text { Chamaecyparis pisifera (Siebold \& } \\
\text { Zucc.) Endl. }\end{array}$ & 116 & 127 & 94.9 & 135 & \\
\hline Cyatheaceae & SCBG & $\begin{array}{l}\text { Sphaeropteris lepifera (Hook.) R. M. } \\
\text { Tryon }\end{array}$ & 103 & 126 & 76.0 & 117 & \\
\hline Cyperaceae & TSUK & Carex oahuensis Hillebr. & 57.6 & 60.2 & 57.9 & 75.3 & $*$ \\
\hline Daphniphyllaceae & KUMN & $\begin{array}{l}\text { Daphniphyllum longeracemosum } \\
\text { Rosenth. }\end{array}$ & 79.4 & 91.4 & 68.4 & 93.8 & \\
\hline Dilleniaceae & SCBG & Dillenia turbinata Finet \& Gagnep. & 60.2 & 113 & 26.7 & 69.9 & $*$ \\
\hline \multirow[t]{2}{*}{ Dipterocarpaceae } & SCBG & Hopea chinensis (Merr.) Hand.-Mazz. & 59.4 & 56.8 & 26.3 & 54.9 & $*$ \\
\hline & SCAU & Hopea hainanensis Merr. \& Chun & 119 & 136 & 60.1 & 107 & \\
\hline Dryopteridaceae & WHUN & Cyrtomium yamamotoi Tagawa & 94.3 & 133 & 59.8 & 90.8 & \\
\hline Ebenaceae & TUAT & Diospyros kaki L. f. & 108 & 119 & 69.5 & 89.9 & \\
\hline \multirow[t]{2}{*}{ Elaeocarpaceae } & SCAU & Elaeocarpus apiculatus Mast. & 94.6 & 117 & 23.5 & 43.9 & \\
\hline & KUMN & $\begin{array}{l}\text { Sloanea hemsleyana Rehder \& E. H. } \\
\text { Wilson }\end{array}$ & 96.9 & 98.3 & 48.3 & 41.5 & \\
\hline \multirow[t]{2}{*}{ Ericaceae } & TUAT & Rhododendron kaempferi Planch. & 61.6 & 85.9 & 31.0 & 65.3 & \\
\hline & TSUK & Pieris japonica D. Don ex G. Don & 114 & 148 & 105 & 154 & \\
\hline Escalloniaceae & KUMN & Itea yunnanensis Franch. & 86.2 & 124 & 64.1 & 110 & \\
\hline \multirow[t]{5}{*}{ Euphorbiaceae } & SCBG & $\begin{array}{l}\text { Bischofia polycarpa (H.Lév.) Airy } \\
\text { Shaw }\end{array}$ & 34.8 & 80.6 & 12.2 & 31.1 & $* * *$ \\
\hline & SCBG & Sapium biglandulosum Müll.Arg. & 44.4 & 52.6 & 17.8 & 27.5 & $* *$ \\
\hline & WHUN & Excoecaria acerifolia Didr. & 45.1 & 91.0 & 16.8 & 45.2 & $* *$ \\
\hline & WHUN & Excoecaria cochinchinensis Lour. & 73.7 & 129 & 63.1 & 97.6 & \\
\hline & SCAU & Bridelia tomentosa Blume & 86.2 & 179 & 20.8 & 82.5 & \\
\hline \multirow[t]{3}{*}{ Fabaceae } & SCBG & Cassia siamea Lam. & 40.6 & 66.7 & 14.6 & 63.7 & $* *$ \\
\hline & SCBG & Erythrophleum fordii Oliv. & 42.4 & 56.1 & 37.9 & 100 & $* *$ \\
\hline & TSUK & Crotalaria sessiliflora $\mathrm{L}$. & 53.6 & 88.9 & 20.8 & 55.9 & $*$ \\
\hline
\end{tabular}




\begin{tabular}{|c|c|c|c|c|c|c|c|}
\hline \multirow{3}{*}{ Plant families } & \multirow{3}{*}{ POC } & \multirow{3}{*}{ Scientific Name } & \multicolumn{4}{|c|}{ Dry leaf content $\left(10 \mathrm{ml} \mathrm{agar}^{-1}\right)$} & \multirow{3}{*}{ Criteria } \\
\hline & & & \multicolumn{2}{|c|}{$10 \mathrm{mg}$} & \multicolumn{2}{|c|}{$50 \mathrm{mg}$} & \\
\hline & & & $\mathrm{R} \%$ & $\mathrm{H} \%$ & $\mathrm{R} \%$ & $\mathrm{H} \%$ & \\
\hline \multirow[t]{8}{*}{ Fabaceae } & SCBG & Pongamia pinnata (L.) Pierre & 55.4 & 98.2 & 32.3 & 100 & $*$ \\
\hline & WHUN & Wisteria sinensis (Sims) DC. & 56.5 & 82.7 & 67.0 & 87.9 & $*$ \\
\hline & TUAT & Styphnolobium japonicum (L.) Schott & 59.7 & 115 & 26.5 & 84.6 & $*$ \\
\hline & SCBG & Sindora tonkinensis A. Chev. & 73.2 & 114 & 54.8 & 115 & \\
\hline & SCBG & Saraca dives Pierre & 60.6 & 111 & 49.5 & 107 & $*$ \\
\hline & SCBG & Pithecellobium lucidum Benth. & 64.6 & 135 & 47.5 & 143 & \\
\hline & SCBG & Bauhinia blakeana Dunn & 65.1 & 74.8 & 33.5 & 85.0 & \\
\hline & TSUK & Macroptilium atropurpureum (L.) Urb. & 99.5 & 107 & 34.2 & 100 & \\
\hline \multirow[t]{8}{*}{ Fagaceae } & TUAT & Quercus myrsinifolia Blume & 75.4 & 106 & 93.6 & 122 & \\
\hline & TUAT & Quercus glauca Thunb. & 80.6 & 109 & 45.2 & 95.9 & \\
\hline & TUAT & Lithocarpus glaber Nakai & 81.1 & 108 & 62.3 & 131 & \\
\hline & TUAT & Quercus gilva Blume & 82.4 & 90.1 & 68.5 & 81.1 & \\
\hline & TUAT & Lithocarpus edulis Nakai & 84.9 & 105 & 61.4 & 114 & \\
\hline & TUAT & Quercus serrata Murray & 86.3 & 104 & 54.8 & 107 & \\
\hline & SCAU & Lithocarpus glaber Nakai & 111 & 153 & 74.3 & 132 & \\
\hline & TUAT & Quercus acutissima Carruth. & 95.1 & 124 & 64.0 & 121 & \\
\hline \multirow[t]{2}{*}{ Ginkgoaceae } & TUAT & Ginkgo biloba $\mathrm{L}$ & 80.8 & 108 & 25.6 & 79.2 & \\
\hline & TUAT & Ginkgo biloba L. (Fruit) & 83.5 & 106 & 30.1 & 69.4 & \\
\hline \multirow[t]{3}{*}{ Hamamelidaceae } & KUMN & Loropetalum chinense Oliv. & 61.6 & 98.7 & 31.1 & 66.7 & \\
\hline & SCBG & Altingia chinensis Oliv. ex Hance & 73.7 & 105 & 57.1 & 119 & \\
\hline & TUAT & Hamamelis japonica Siebold \& Zucc. & 76.9 & 115 & 57.4 & 103 & \\
\hline Hemerocallidaceae & TSUK & Hemerocallis fulva $\mathrm{L}$. & 35.5 & 69.9 & 36.2 & 73.2 & $* * *$ \\
\hline Hippocastanaceae & TUAT & Aesculus turbinata Blume & 71.0 & 96.3 & 61.7 & 109 & \\
\hline Hydrangeaceae & TSUK & Hydrangea macrophylla (Thunb.) Ser. & 65.9 & 80.1 & 55.9 & 100 & \\
\hline Iridaceae & SCBG & Iris japonica Thunb. & 52.5 & 89.2 & 23.5 & 63.7 & $*$ \\
\hline Juglandaceae & TUAT & Platycarya strobilacea Siebold \& Zucc. & 67.6 & 117 & 38.9 & 86.8 & \\
\hline \multirow[t]{6}{*}{ Lamiaceae } & SCBG & Vitex quinata $\mathrm{F} . \mathrm{N}$. Williams & 60.2 & 88.3 & 23.1 & 65.5 & $*$ \\
\hline & TSUK & Callicarpa japonica Thunb. & 60.4 & 76.7 & 48.0 & 92.8 & $*$ \\
\hline & SCBG & Epimeredi indica (L.) Rothm. & 69.2 & 119 & 44.4 & 81.0 & \\
\hline & TSUK & Scutellaria baicalensis Georgi & 72.2 & 108 & 41.9 & 97.7 & \\
\hline & KUMN & Callicarpa macrophylla Vahl & 87.4 & 113 & 62.6 & 99.1 & \\
\hline & TSUK & Keiskea japonica Miq. & 107 & 159 & 41.8 & 123 & \\
\hline \multirow[t]{2}{*}{ Lardizabalaceae } & TSUK & $\begin{array}{l}\text { Akebia quinata (Thumb. ex Houtt.) } \\
\text { Decne. }\end{array}$ & 65.7 & 104 & 55.2 & 86.4 & \\
\hline & TSUK & Stauntonia hexaphylla Decne. & 94.7 & 120 & 66.1 & 147 & \\
\hline \multirow[t]{8}{*}{ Lauraceae } & WHUN & Lindera fragrans Oliv. & 62.2 & 95.3 & 41.7 & 87.3 & \\
\hline & SCBG & Machilus oculodracontis Chun & 69.2 & 107 & 30.3 & 68.9 & \\
\hline & WHUN & Cinnamomum osmophloeum Keneh. & 76.0 & 110 & 42.7 & 62.6 & \\
\hline & SCAU & $\begin{array}{l}\text { Cinnamomum burmannii (Nees \& T. } \\
\text { Nees) Blume }\end{array}$ & 88.6 & 108 & 45.9 & 91.2 & \\
\hline & TUAT & Laurus nobilis L. & 68.7 & 118 & 42.3 & 77.1 & \\
\hline & SCBG & $\begin{array}{l}\text { Cinnamomum porrectum (Roxb.) } \\
\text { Kosterm. }\end{array}$ & 73.2 & 96.5 & 70.7 & 112 & \\
\hline & TUAT & Cinnamomum camphora (L.) J. Presl & 82.2 & 108 & 54.7 & 121 & \\
\hline & SCBG & Litsea verticillata Hance & 74.3 & 107 & 52.0 & 96.5 & \\
\hline Liliaceae & SCBG & Tupistra glandistigma Wang et Tang & 65.7 & 92.1 & 35.3 & 72.5 & \\
\hline Davalliaceae & KUMN & Nephrolepis cordifolia (L.) K. Presl & 88.3 & 122 & 65.1 & 120 & \\
\hline \multirow[t]{2}{*}{ Lythraceae } & SCBG & Lagerstroemia speciosa (L.) Pers. & 81.7 & 125 & 17.5 & 63.9 & \\
\hline & TUAT & Lagerstroemia indica L. & 85.8 & 117 & 36.7 & 91.0 & \\
\hline \multirow[t]{6}{*}{ Magnoliaceae } & SCBG & Magnolia liliiflora Desr. & 35.4 & 64.9 & 26.3 & 56.9 & *** \\
\hline & SCBG & $\begin{array}{l}\text { Manglietia lucida B. L. Chen \& S. C. } \\
\text { Yang }\end{array}$ & 64.8 & 82.0 & 35.9 & 95.6 & \\
\hline & SCBG & $\begin{array}{l}\text { Magnolia sirindhorniae Noot. \& } \\
\text { Chalermglin }\end{array}$ & 69.7 & 100 & 23.8 & 45.1 & \\
\hline & SCBG & Manglietia insignis Blume & 71.3 & 123 & 37.6 & 97.1 & \\
\hline & TUAT & Magnolia obovata Thunb. & 73.1 & 105 & 44.0 & 73.4 & \\
\hline & TUAT & Magnolia grandiflora L. & 84.7 & 100 & 81.3 & 121 & \\
\hline
\end{tabular}




\begin{tabular}{|c|c|c|c|c|c|c|c|}
\hline \multirow{3}{*}{ Plant families } & \multirow{3}{*}{ POC } & \multirow{3}{*}{ Scientific Name } & \multicolumn{4}{|c|}{ Dry leaf content $\left(10 \mathrm{ml} \mathrm{agar}^{-1}\right)$} & \multirow{3}{*}{ Criteria } \\
\hline & & & \multicolumn{2}{|c|}{$10 \mathrm{mg}$} & \multicolumn{2}{|c|}{$50 \mathrm{mg}$} & \\
\hline & & & $\mathrm{R} \%$ & $\mathrm{H} \%$ & $\mathrm{R} \%$ & $\mathrm{H} \%$ & \\
\hline \multirow[t]{10}{*}{ Magnoliaceae } & TUAT & Liriodendron tulipifera $\mathrm{L}$. & 85.8 & 106 & 45.3 & 92.2 & \\
\hline & SCAU & Michelia balansae Dandy & 102 & 145 & 61.7 & 147 & \\
\hline & SCAU & $\begin{array}{l}\text { Michelia sphaerantha C.Y. Wu ex Z.S. } \\
\text { Yue }\end{array}$ & 88.0 & 104 & 61.2 & 94.7 & \\
\hline & SCAU & $\begin{array}{l}\text { Michelia fadouensis D. X. Li \& Y. W. } \\
\text { Law }\end{array}$ & 104 & 172 & 76.5 & 121 & \\
\hline & TUAT & Magnolia Kobus DC. & 105 & 164 & 61.9 & 113 & \\
\hline & KUMN & Michelia figo (Lour.) Spreng. & 59.3 & 96.6 & 31.1 & 64.6 & $*$ \\
\hline & KUMN & $\begin{array}{l}\text { Michelia yunnanensis Franch. ex Finet } \\
\text { \& Gagnep. }\end{array}$ & 80.1 & 88.8 & 60.3 & 73.0 & \\
\hline & SCAU & Michelia alba DC. & 78.2 & 80.7 & 75.4 & 78.4 & \\
\hline & SCAU & Manglietia fordiana Oliv. & 102 & 160 & 50.3 & 130 & \\
\hline & SCAU & Tsoongiodendron odorum Chun & 123 & 149 & 84.7 & 147 & \\
\hline \multirow[t]{2}{*}{ Malvaceae } & SCBG & Hibiscus syriacus L. & 14.6 & 75.4 & 5.6 & 32.8 & $* * * * *$ \\
\hline & SCBG & Hibiscus mutabilis L. & 61.6 & 146 & 24.2 & 75.9 & \\
\hline Meliaceae & SCBG & Aglaia odorata Lour. & 92.4 & 118 & 51.2 & 96.5 & \\
\hline \multirow[t]{7}{*}{ Moraceae } & TUAT & Morus bombycis Koidz. & 49.1 & 106 & 15.3 & 70.8 & $* *$ \\
\hline & SCBG & Ficus drupacea Thunb. & 49.5 & 96.5 & 23.2 & 87.9 & $* *$ \\
\hline & SCBG & Ficus fistulosa Reinw. ex Blume & 59.0 & 91.9 & 32.4 & 96.5 & $*$ \\
\hline & SCBG & Ficus benjamina $\mathrm{L}$. & 72.4 & 128 & 31.0 & 66.4 & \\
\hline & SCAU & Ficus lacor Buch.-Ham. & 86.2 & 127 & 27.9 & 86.0 & \\
\hline & SCBG & Ficus annulata Blume & 92.4 & 184 & 49.5 & 160 & \\
\hline & WHUN & Ficus microcarpa L. f. & 72.8 & 117 & 40.4 & 111 & \\
\hline Myricaceae & TSUK & Myrica rubra (Lour.) Siebold \& Zucc. & 84.5 & 116 & 67.0 & 110 & \\
\hline \multirow[t]{2}{*}{ Myrsinaceae } & TSUK & Ardisia crenata Roxb. & 60.1 & 84.7 & 35.6 & 62.7 & $*$ \\
\hline & WHUN & $\begin{array}{l}\text { Rapanea neriifolia (Siebold \& Zucc.) } \\
\text { Mez }\end{array}$ & 84.6 & 166 & 43.1 & 104 & \\
\hline Myrtaceae & SCBG & Eugenia javanica Lam. & 83.3 & 112 & 45.5 & 106 & \\
\hline \multirow{7}{*}{ Oleaceae } & SCBG & Osmanthus matsumuranus Hayata & 52.0 & 104 & 27.8 & 89.7 & $*$ \\
\hline & TUAT & Ligustrum lucidum W. T. Aiton & 80.2 & 103 & 47.3 & 103 & \\
\hline & KUMN & $\begin{array}{l}\text { Ligustrum compactum (Wall. ex G. } \\
\text { Don) Hook. f. \& Thomson ex Brandis }\end{array}$ & 83.3 & 97.8 & 72.9 & 83.5 & \\
\hline & SCBG & Osmanthus fragrans Lour. & 84.6 & 149 & 65.4 & 121 & \\
\hline & TUAT & Fraxinus longicuspis Siebold \& Zucc. & 86.1 & 90.9 & 81.5 & 125 & \\
\hline & TUAT & Osmanthus fragrans Lour. & 88.7 & 103 & 89.6 & 128 & \\
\hline & WHUN & Olea europaea L. & 86.6 & 93.3 & 82.4 & 71.3 & \\
\hline Orchidaceae & TSUK & Epipactis thunbergii A. Gray & 61.1 & 78.9 & 67.7 & 71.0 & \\
\hline Oxalidaceae & SCBG & Averrhoa carambola $\mathrm{L}$. & 67.0 & 115 & 22.8 & 73.5 & \\
\hline Papaveraceae & KUMN & Corydalis taliensis Franch. & 55.9 & 70.0 & 26.3 & 37.6 & $*$ \\
\hline \multirow[t]{2}{*}{ Pinaceae } & TUAT & Pinus parviflora Siebold \& Zucc. & 73.2 & 104 & 46.2 & 88.4 & \\
\hline & TUAT & Pinus thunbergii Parl. & 82.5 & 81.5 & 39.6 & 57.5 & \\
\hline \multirow[t]{2}{*}{ Piperaceae } & WHUN & Piper sarmentosum Roxb. & 39.9 & 94.8 & 14.5 & 65.8 & $* *$ \\
\hline & SCBG & Piper sarmentosum Roxb. & 64.6 & 105 & 49.5 & 95.6 & \\
\hline Platanaceae & TUAT & Platanus orientalis L. & 90.2 & 116 & 71.9 & 127 & \\
\hline \multirow[t]{2}{*}{ Poaceae } & WHUN & $\begin{array}{l}\text { Indocalamus tessellatus (Munro) } \\
\text { Keng f. }\end{array}$ & 56.2 & 91.3 & 34.0 & 89.7 & $*$ \\
\hline & TSUK & Miscanthus condensatus Hack. & 57.9 & 82.2 & 46.2 & 88.2 & $*$ \\
\hline \multirow[t]{2}{*}{ Podocarpaceae } & SCAU & Nageia nagi Britton \& P.Wilson & 102 & 143 & 77.6 & 89.5 & \\
\hline & SCAU & Podocarpus fleuryi Hickel & 115 & 117 & 55.2 & 68.4 & \\
\hline Primulaceae & TSUK & Lysimachia daphnoides Hillebr. & 74.3 & 101 & 16.3 & 47.4 & \\
\hline \multirow[t]{2}{*}{ Ranunculaceae } & KUMN & Anemone vitifolia Buch.-Ham. ex DC. & 54.1 & 80.9 & 38.9 & 59.1 & $*$ \\
\hline & TSUK & Caltha palustris $\mathrm{L}$. & 60.5 & 111 & 37.4 & 104 & $*$ \\
\hline \multirow[t]{2}{*}{ Rhamnaceae } & TSUK & Ziziphus jujuba Mill. & 59.5 & 94.4 & 23.1 & 59.1 & $*$ \\
\hline & SCBG & Sageretia thea (Osbeck) M. C. Johnst. & 80.8 & 124 & 48.4 & 104 & \\
\hline Rosaceae & KUMN & Photinia glabra (Thunb.) Maxim. & 0.0 & 0.0 & 0.0 & 0.0 & $* * * * *$ \\
\hline & TSUK & Amygdalus persica $\mathrm{L}$. & 17.1 & 29.1 & 13.1 & 26.8 & $* * * * *$ \\
\hline & TUAT & Prunus buergeriana Miq. & 51.2 & 112 & 17.2 & 81.8 & $*$ \\
\hline & TUAT & Cerasus jamasakura (Koidz.) H. Ohba & 57.2 & 89.0 & 57.3 & 88.1 & $*$ \\
\hline
\end{tabular}




\begin{tabular}{|c|c|c|c|c|c|c|c|}
\hline \multirow{3}{*}{ Plant families } & \multirow{3}{*}{ POC } & \multirow{3}{*}{ Scientific Name } & \multicolumn{4}{|c|}{ Dry leaf content $\left(10 \mathrm{ml} \mathrm{agar}^{-1}\right)$} & \multirow{3}{*}{ Criteria } \\
\hline & & & \multicolumn{2}{|c|}{$10 \mathrm{mg}$} & \multicolumn{2}{|c|}{$50 \mathrm{mg}$} & \\
\hline & & & $\mathrm{R} \%$ & $\mathrm{H} \%$ & $\mathrm{R} \%$ & $\mathrm{H} \%$ & \\
\hline \multirow[t]{7}{*}{ Rosaceae } & TUAT & Prunus yedoensis Matsum. & 77.1 & 113 & 56.4 & 109 & \\
\hline & KUMN & $\begin{array}{l}\text { Laurocerasus undulata (Buch.-Ham. ex } \\
\text { D. Don) M. Roem. }\end{array}$ & 59.0 & 82.0 & 33.7 & 60.0 & $*$ \\
\hline & KUMN & Prinsepia utilis Royle & 77.6 & 94.4 & 61.0 & 82.6 & \\
\hline & TUAT & Eriobotrya japonica (Thunb.) Lindl. & 87.6 & 134 & 72.0 & 135 & \\
\hline & TUAT & Prunus lannesiana E. H. Wilson & 88.0 & 103 & 50.2 & 89.0 & \\
\hline & TSUK & Spiraea japonica L.f. & 111 & 166 & 66.2 & 149 & \\
\hline & TUAT & Cerasus speciosa (Koidz.) H. Ohba & 75.9 & 99.2 & 41.3 & 97.6 & \\
\hline \multirow[t]{2}{*}{ Rubiaceae } & SCBG & Gardenia sootepensis Hutch. & 70.1 & 91.0 & 35.9 & 79.6 & \\
\hline & SCBG & Psychotria rubra Poir. & 77.3 & 118 & 37.9 & 89.7 & \\
\hline \multirow[t]{5}{*}{ Rutaceae } & SCBG & Acronychia pedunculata Miq. & 39.4 & 84.2 & 28.8 & 75.9 & $* * *$ \\
\hline & SCBG & Atalantia buxifolia (Poir.) Oliv. & 46.0 & 97.5 & 49.0 & 119 & $* *$ \\
\hline & TSUK & Phellodendron amurense Rupr. & 53.1 & 88.3 & 20.7 & 67.8 & $*$ \\
\hline & SCBG & Clausena lansium Skeels & 66.3 & 118 & 25.1 & 86.4 & \\
\hline & TUAT & Citrus junos Siebold ex Tanaka & 84.0 & 109 & 49.8 & 114 & \\
\hline \multirow[t]{3}{*}{ Sapindaceae } & SCBG & Dimocarpus longan Lour. & 52.9 & 108 & 23.8 & 95.6 & $*$ \\
\hline & TUAT & Sapindus mukorossi Gaertn. & 60.0 & 104 & 25.6 & 53.4 & $*$ \\
\hline & WHUN & Litchi chinensis Sonn. & 83.8 & 117 & 58.0 & 109 & \\
\hline Sapotaceae & SCBG & Madhuca pasquieri H. J. Lam & 41.8 & 74.8 & 18.5 & 81.4 & $* *$ \\
\hline Saxifragaceae & TSUK & Astilbe microphylla Knoll & 70.6 & 108 & 36.5 & 92.5 & \\
\hline Schisandraceae & SCBG & Kadsura coccinea (Lem.) A. C. Sm. & 44.8 & 90.1 & 15.7 & 47.8 & $* *$ \\
\hline \multirow[t]{2}{*}{ Solanaceae } & KUMN & $\begin{array}{l}\text { Anisodus acutangulus C. Y. Wu \& C. } \\
\text { Chen }\end{array}$ & 44.9 & 116 & 25.5 & 106 & $* *$ \\
\hline & SCBG & Datura metel L. & 63.0 & 122 & 39.2 & 133 & \\
\hline Sterculiaceae & SCAU & Pterospermum heterophyllum Hance & 113 & 153 & 65.6 & 132 & \\
\hline Styracaceae & TUAT & Styrax japonica Siebold \& Zucc. & 66.9 & 91.4 & 49.4 & 80.3 & \\
\hline Symplocaceae & SCBG & $\begin{array}{l}\text { Symplocos cochinchinensis (Lour.) S. } \\
\text { Moore }\end{array}$ & 47.9 & 94.2 & 18.5 & 41.7 & $* *$ \\
\hline \multirow[t]{3}{*}{ Taxaceae } & KUMN & Taxus wallichiana Zucc. & 35.1 & 98.2 & 36.0 & 54.8 & $* * *$ \\
\hline & WHUN & Taxus chinensis Roxb. & 56.2 & 128 & 35.4 & 102 & $*$ \\
\hline & TUAT & Torreya nucifera Siebold \& Zucc. & 98.5 & 128 & 73.9 & 104 & \\
\hline \multirow[t]{4}{*}{ Taxodiaceae } & TUAT & $\begin{array}{l}\text { Metasequoia glyptostroboides } \mathrm{Hu} \& \mathrm{~W} \text {. } \\
\text { C. Cheng }\end{array}$ & 53.7 & 116 & 14.8 & 92.4 & $*$ \\
\hline & TSUK & $\begin{array}{l}\text { Metasequoia glyptostroboides } \mathrm{Hu} \& \mathrm{~W} \text {. } \\
\text { C. Cheng }\end{array}$ & 56.7 & 78.6 & 29.4 & 66.0 & $*$ \\
\hline & TUAT & Taxodium distichum (L.) Rich. & 82.9 & 117 & 33.0 & 78.5 & \\
\hline & TUAT & Cryptomeria japonica D. Don & 107 & 121 & 88.9 & 117 & \\
\hline \multirow[t]{5}{*}{ Theaceae } & SCBG & Camellia oleifera C. Abel & 50.4 & 80.7 & 14.1 & 45.8 & $*$ \\
\hline & TSUK & Camellia sasanqua Thunb. & 71.0 & 75.6 & 26.5 & 32.3 & \\
\hline & TUAT & $\begin{array}{l}\text { Ternstroemia gymnanthera (Wight \& } \\
\text { Am.) Bedd. }\end{array}$ & 75.6 & 107 & 69.6 & 115 & \\
\hline & SCAU & Schima spp & 77.2 & 166 & 35.5 & 119 & \\
\hline & TUAT & Camellia sasanqua Thunb. & 83.6 & 110 & 56.1 & 78.0 & \\
\hline Thymelaeaceae & KUMN & Daphne papyracea Wall. ex Steud. & 41.5 & 111 & 24.8 & 66.1 & $* *$ \\
\hline Tropaeolaceae & TSUK & Tropaeolum majus $\mathrm{L}$. & 60.0 & 116 & 31.2 & 73.2 & $*$ \\
\hline \multirow[t]{2}{*}{ Ulmaceae } & TUAT & Aphananthe aspera Planch. & 65.2 & 119 & 38.4 & 97.2 & \\
\hline & TUAT & Zelkova serrata (Thunb.) Makino & 71.8 & 138 & 35.4 & 129 & \\
\hline Ulmaceae & TUAT & Celtis sinensis Pers. & 88.3 & 112 & 45.6 & 113 & \\
\hline Urticaceae & TSUK & Boehmeria tenuifolia Satake & 70.2 & 113 & 30.4 & 108 & \\
\hline Valerianaceae & TSUK & Patrinia villosa Juss. & 86.9 & 118 & 57.0 & 112 & \\
\hline Verbenaceae & KUMN & Duranta erecta $\mathrm{L}$. & 43.3 & 77.6 & 7.7 & 26.2 & $* *$ \\
\hline \multirow[t]{3}{*}{ Zingiberaceae } & WHUN & $\begin{array}{l}\text { Hedychium coccineum Buch.-Ham. ex } \\
\text { Sm. }\end{array}$ & 66.0 & 100 & 31.1 & 80.0 & \\
\hline & WHUN & Alpinia oxyphylla Miq. & 54.3 & 109 & 24.7 & 70.9 & $*$ \\
\hline & WHUN & Amomum tsaoko Crevost \& Lemarie & 67.9 & 149 & 21.8 & 90.4 & \\
\hline
\end{tabular}


Table 2: Determination of allelopathic activity by volatile compounds in some plant species in the Sino-Japanese Region using the dish pack method

\begin{tabular}{|c|c|c|c|c|c|c|c|}
\hline \multirow{3}{*}{ Plant families } & \multirow{3}{*}{ POC } & \multirow{3}{*}{ Scientific name } & \multicolumn{4}{|c|}{ Inhibition activity } & \multirow{3}{*}{ Criteria } \\
\hline & & & \multicolumn{2}{|c|}{$\begin{array}{l}\text { Average for } \\
\text { whole wells }\end{array}$} & \multicolumn{2}{|c|}{$\begin{array}{l}\text { Average at } \\
41 \mathrm{~mm}\end{array}$} & \\
\hline & & & $\mathrm{R} \%$ & $\mathrm{H} \%$ & $\mathrm{R} \%$ & $\mathrm{H} \%$ & \\
\hline \multirow[t]{5}{*}{ Aceraceae } & TUAT & Acer cissifolium K. Koch & 22.9 & 33.6 & 33.0 & 35.7 & \\
\hline & TUAT & Acer palmatum Thunb. & 12.7 & 8.0 & 23.5 & 21.1 & \\
\hline & TUAT & Acer diabolicum Blume ex K. Koch & 11.9 & -0.8 & 13.1 & -8.4 & \\
\hline & TUAT & Acer buergerianum Miq. & -2.8 & -9.2 & 3.4 & -14.6 & \\
\hline & TUAT & Acer mono Maxim. & -9.8 & -6.6 & 14.3 & -0.4 & + \\
\hline Amaryllidaceae & WHBG & Lycoris radiata Herb. & 23.1 & 20.2 & 32.6 & 31.5 & \\
\hline Annonaceae & SCAU & Polyalthia longifolia (Soon.) Thwaites & -7.6 & 0.5 & -18.6 & -6.6 & + \\
\hline Altingiaceae & TUAT & Liquidambar styraciflua $\mathrm{L}$. & 61.4 & 2.8 & 61 & 3.1 & $* * *$ \\
\hline Arecaceae & SCAU & Areca triandra Roxb. ex Buch.-Ham. & 21.4 & 21.7 & 30.8 & 29.9 & \\
\hline Asparagaceae & TKBG & Hosta sieboldiana (Hook.) Engl. & 21.7 & 11.3 & 24.5 & 13.3 & \\
\hline Asteraceae & TKBG & Ligularia fischeri Turcz. & 10.3 & 8.9 & 11.0 & 10.1 & \\
\hline Berberidaceae & TKBG & Nandina domestica Thunb. & 22.1 & 13.1 & 26.2 & 25.4 & \\
\hline Betulaceae & TUAT & Betula platyphylla Sukaczev & -2.3 & -10.9 & -1.8 & -12.4 & \\
\hline Bombacaceae & SCBG & Bombax malabaricum DC. & -3.3 & -15.3 & -3.9 & -25.7 & \\
\hline Caprifoliaceae & TUAT & Viburnum odoratissimum Ker Gawl. & 2.1 & -2.7 & 3.5 & -1.7 & \\
\hline Cephalotaxaceae & WHBG & Cephalotaxus fortunei Hook. & -10.3 & -12.9 & -1.9 & -1.1 & ++ \\
\hline Cercidiphyllaceae & TUAT & $\begin{array}{l}\text { Cercidiphyllum japonicum Siebold \& } \\
\text { Zucc. }\end{array}$ & 27.0 & 9.1 & 31.8 & 6.7 & \\
\hline Cornaceae & TUAT & $\begin{array}{l}\text { Benthamidia japonica (Siebold \& } \\
\text { Zucc.) H. Hara }\end{array}$ & 13.9 & -24.6 & 6.7 & -37.7 & \\
\hline Cupressaceae & TUAT & Juniperus chinensis L. & 24.5 & 18.5 & 34.0 & 15.1 & \\
\hline Daphniphyllaceae & KMBG & $\begin{array}{l}\text { Daphniphyllum longeracemosum } \\
\text { Rosenth. }\end{array}$ & 3.5 & -6.8 & 8.2 & 2.6 & \\
\hline Dipterocarpaceae & SCAU & Hopea hainanensis Merr. \& Chun & 3.9 & 1.5 & 8.1 & 2.2 & \\
\hline Ebenaceae & TUAT & Diospyros kaki L.f. & -11.0 & -10.9 & -15.7 & -17 & + \\
\hline Elaeocarpaceae & SCAU & Elaeocarpus apiculatus Mast. & 20.0 & 21.1 & 23.2 & 21.1 & \\
\hline \multirow[t]{2}{*}{ Fabaceae } & SCBG & Saraca dives Pierre & 2.4 & 4.2 & 5.4 & 5.6 & \\
\hline & WHBG & Wisteria sinensis (Sims) DC. & 0.4 & -1.1 & 4.5 & 0.3 & \\
\hline \multirow[t]{3}{*}{ Fagaceae } & TUAT & Lithocarpus edulis Nakai & 22.7 & 1.9 & 27.0 & 13.9 & \\
\hline & TUAT & Quercus serrata Murray & 4.5 & -5.3 & 8.9 & 5.5 & \\
\hline & TUAT & Quercus gilva Blume & -16.1 & -6.4 & -11.3 & -5.7 & ++ \\
\hline Ginkgoaceae & TUAT & Ginkgo biloba $\mathrm{L}$. & 12.9 & -9.2 & 13.2 & -12.9 & \\
\hline Hamamelidaceae & TUAT & Hamamelis japonica Siebold \& Zucc. & 11.3 & 7.8 & 4.0 & 3.2 & \\
\hline Hippocastanaceae & TUAT & Aesculus turbinata Blume & -33.0 & -7.9 & -23.1 & -7.0 & +++ \\
\hline Juglandaceae & TUAT & Platycarya strobilacea Siebold \& Zucc. & 34.9 & 17.9 & 45.0 & 9.7 & $*$ \\
\hline Lamiaceae & KMBG & Callicarpa macrophylla Vahl. & 1.3 & 1.6 & 5.1 & 1.9 & \\
\hline \multirow[t]{2}{*}{ Lauraceae } & TUAT & Cinnamomum camphora (L.) J. Presl & 50.2 & 59.9 & 43.7 & 63.0 & $* * *$ \\
\hline & TUAT & Laurus nobilis L. & 3.6 & 5.0 & 7.0 & 6.6 & \\
\hline Liliaceae & SCBG & Tupistra glandistigma Wang et Tang & 1.8 & 1.6 & 4.1 & 3.0 & \\
\hline Lythraceae & SCBG & Lagerstroemia speciosa (L.) Pers. & 3.6 & 0.9 & 5.2 & 1.6 & \\
\hline \multirow[t]{9}{*}{ Magnoliaceae } & TUAT & Liriodendron tulipifera $\mathrm{L}$. & 19.1 & 7.8 & 16.1 & 13.6 & \\
\hline & SCAU & Michelia balansae Dandy & 12.8 & -40.5 & 21.6 & -32.9 & \\
\hline & SCBG & Magnolia liliiflora Ders. & 10.7 & 1.6 & 21.1 & 7.9 & \\
\hline & SCAU & Manglietia fordiana Oliv. & 10.1 & 0.0 & 19.1 & -1.3 & \\
\hline & SCAU & Tsoongiodendron odorum Chun & 7.2 & 0.8 & 9.2 & -0.7 & \\
\hline & TUAT & Magnolia grandiflora L. & 4.8 & -2.2 & 2.0 & -12.3 & \\
\hline & SCAU & $\begin{array}{l}\text { Magnolia megaphylla (Hu \& W. C. } \\
\text { Cheng) V. S. Kumar }\end{array}$ & 1.4 & 2.6 & 4.1 & 0.0 & \\
\hline & TUAT & Magnolia kobus DC. & -3.1 & 2.1 & 2.8 & 8.7 & \\
\hline & TUAT & Magnolia obovata Thunb. & -8.2 & -6.8 & 0.3 & -1.1 & + \\
\hline Moraceae & TKBG & Morus alba $\mathrm{L}$. & 27.1 & 17.0 & 29.7 & 19.3 & \\
\hline Myrtaceae & SCBG & Eugenia javanica Lam. & 12.0 & 9.0 & 23.6 & 18.0 & \\
\hline \multirow[t]{2}{*}{ Oleaceae } & TUAT & Fraxinus longicuspis Siebold \& Zucc. & -10.0 & -4.1 & -4.9 & 2.3 & + \\
\hline & TUAT & Ligustrum lucidum W. T. Aiton & 10.9 & 6.7 & 12.5 & 7.9 & \\
\hline Pinaceae & TUAT & Pinus parviflora Siebold \& Zucc. & 35.5 & 29.9 & 35.7 & 29.8 & $*$ \\
\hline
\end{tabular}




\begin{tabular}{|c|c|c|c|c|c|c|c|}
\hline \multirow{3}{*}{ Plant families } & \multirow{3}{*}{ POC } & \multirow{3}{*}{ Scientific name } & \multicolumn{4}{|c|}{ Inhibition activity } & \multirow{3}{*}{ Criteria } \\
\hline & & & \multicolumn{2}{|c|}{$\begin{array}{l}\text { Average for } \\
\text { whole wells }\end{array}$} & \multicolumn{2}{|c|}{$\begin{array}{l}\text { Average at } \\
41 \mathrm{~mm}\end{array}$} & \\
\hline & & & $\mathrm{R} \%$ & $\mathrm{H} \%$ & $\mathrm{R} \%$ & $\mathrm{H} \%$ & \\
\hline Platanaceae & TUAT & Platanus orientalis L. & 1.8 & -4.1 & -1.1 & -2.5 & \\
\hline Podocarpaceae & SCAU & Podocarpus fleuryi Hickel & 4.9 & 8.5 & 10.4 & 15.3 & \\
\hline \multirow[t]{7}{*}{ Rosaceae } & TUAT & Cerasus speciosa (Koidz.) H. Ohba & 4.6 & -10.3 & 18.2 & -11.2 & \\
\hline & TKBG & Amygdalus persica $\mathrm{L}$. & 36.2 & 28.9 & 41.0 & 44.1 & $*$ \\
\hline & KMBG & Photinia glabra (Thunb.) Maxim. & 90.6 & 86.1 & 95.2 & 90.2 & $* * *$ \\
\hline & TUAT & Prunus yedoensis Matsum. & 13.1 & -1.4 & 24.0 & -3.3 & \\
\hline & TUAT & Prunus jamasakura Siebold ex Koidz. & 11.8 & 9.1 & 13.0 & 14.9 & \\
\hline & TUAT & Prunus lannesiana E. H. Wilson & 4.3 & -4.3 & 7.3 & 7.6 & \\
\hline & TUAT & Prunus buergeriana Miq. & -10.8 & -2.6 & -2.4 & 7.2 & + \\
\hline Rubiaceae & SCBG & Gardenia sootepensis Hutch. & 32.0 & 24.3 & 41.4 & 31.2 & $*$ \\
\hline \multirow[t]{2}{*}{ Sapindaceae } & TUAT & Sapindus mukorossi Gaertn. & 17.9 & 7.1 & 22.1 & 12.9 & \\
\hline & WHBG & Litchi chinensis Sonn. & 12.0 & 0.5 & 21.1 & 7.9 & \\
\hline Schisandraceae & SCBG & Kadsura coccinea (Lem.) A. C. Sm. & 17.1 & 3.7 & 26.7 & 8.7 & \\
\hline Styracaceae & TUAT & Styrax japonica Siebold \& Zucc. & 13.9 & 12.2 & 27.7 & 17.3 & \\
\hline \multirow[t]{2}{*}{ Taxodiaceae } & TUAT & $\begin{array}{l}\text { Metasequoia glyptostroboides } \mathrm{Hu} \& \mathrm{~W} \text {. } \\
\text { C. Cheng }\end{array}$ & 42.0 & 22.5 & 47.3 & 20.5 & $* *$ \\
\hline & TUAT & Sciadopitys verticillata Siebold \& Zucc. & 38.4 & 37.3 & 42.0 & 49.3 & $*$ \\
\hline Ulmaceae & TUAT & Zelkova serrata (Thunb.) Makino & 12.9 & 2.3 & 14.4 & 12.4 & \\
\hline Zingiberaceae & WHBG & Alpinia oxyphylla Miq. & 29.0 & 26.4 & 34.5 & 39.6 & \\
\hline
\end{tabular}

* Criteria $(*),(* *)$, and $(* * *)$ refer to radicle elongation shorter than the mean value plus $1.0(\mathrm{SD}), 1.5(\mathrm{SD})$ and $2(\mathrm{SD})$, that is, SDV $=31,40$, and 50 , respectively. + Criteria $(+),(++)$, and $(+++)$ refer to radicle elongation longer than the mean value minus $1.0(\mathrm{SD}), 1.5(\mathrm{SD})$ and $2(\mathrm{SD})$, that is, $\mathrm{SDV}=$ $-6,-10$, and -25, respectively. POC; Place of Collection. TUAT; Tokyo University of Agriculture and Technology, TKBG; Tsukuba Botanical Gardens, TMBG; Tokyo Medicinal Botanical Garden, WHBG Wuhan Botanical Garden, KMBG; Kunming Botanical Garden, SCBG; South China Botanical Garden, SCUA; South China University of Agriculture.

Another species of interest in this family is Amygdalus persica which is a fruit of ornamental importance. A. persica is native to China where it have been cultivated for centuries [28]. Dried seeds of A. persica have been used in combination with other herbal plants to overcome stroke-induced disability [29], [30]. A. persica have been reported as a non-food biodiesel plant resources based on grey relation analysis with extremely complicated genetic diversity [31]. Glucosid amygdalin and hydrocyanic acid are the principal constituents of A. persica [32]. In the Malvaceae family, species that showed strong inhibition on lettuce radicle elongation was Hibiscus syriacus. Hibiscus syriacus is native to tropical climates, but are grown around the world for medicinal use and aesthetic value. H. syriacus have been used to treat ailments like gastrointestinal disorders, fevers, respiratory disorder as cough, used as emollient [33]. Sporopollenin observed from pollen of $H$. syriacus have a simple aliphatic polymer containing aromatic or conjugated side chains as the main structure [34]. In a screening for lipid peroxidation inhibitors, Yoo et al., [35] isolated three naphthalene compounds: 2,7-dihydroxy-6-methyl-8-methoxy-1-naphthalenecarboxaldehyde, 2-hydroxy-6-hydroxymethyl-7,8dimethoxy-1-naphthalene-carboxaldehyde, and 1-carboxy-2,8-dihydroxy-6- methyl-7-methoxynaphthalenecarbolactone, designed as syriacusins $\mathrm{A}-\mathrm{C}$, from the chloroform extract of the root bark of $H$. syriacus. All the three compounds inhibited lipid peroxidation. Novel cyclic peptide Hibispeptin a $\left(\mathrm{C}_{39} \mathrm{H}_{50} \mathrm{~N}_{6} \mathrm{O}_{8}\right)$ and Hibispeptin $\mathrm{B}\left(\mathrm{C}_{36} \mathrm{H}_{52} \mathrm{~N}_{6} \mathrm{O}_{8}\right)$ have been isolated from the root bark of H. syriacus [36], [37].

In the Boraginaceous family, Cordia dichotoma had the highest inhibition on lettuce radicle elongation. Cordia dichotoma have been listed as non-consensus invasive woody plant in the coastal and dry lowlands in Mauritius [38]. This species have been used traditionally in India to treat ulcerative colitis (UC) and colic pain. Ganjare et al., [39] showed that apigenin isolated from the bark of Cordia dichotoma was responsible for the treatment of UC since it showed significant healing and reduction in inflammation enzymes when screened against UC. Polysaccharide in fruit of Cordia dichotoma is a potential candidate for use as herbal excipient in the formulation of orodispersible tablets [40]. The leaves and bark of Cordia dichotoma have shown high antioxidant, antimicrobial and ant implantation activities [41], [42], and [43]. The leaves have been found to contain querecetin and quecitrin whereas arabinoglucan, Larabinose and D-glucose have been found in the fruits [44].

Another species that showed strong inhibitory potential through the volatiles released is Liquidambar styraciflua (also known as sweetgum) of the family Altingiaceae. The major components of the leaf oil were reported to be styrene, $d-$ limonene, $\alpha$-pinene and $\beta$-pinene, and that of the stem oil were germacrine $\mathrm{D}, \alpha$-cadinol, $d$-limonene, $\alpha$-pinene, and $\beta$ pinene [45], [46]. These essential oils showed anti-inflammatory activity with low cytotoxicity thus backing its traditional use in treating inflammation. The emission of isoprene from sweetgum has been shown to be dependent on light and severe drought conditions [47], [48]. Some influenza viruses and the virus responsible for H1N1 are susceptible to the antiviral Tamiflu ${ }^{\circledR}$. Shikimic acid is a precursor of oseltamivir phosphate which is the key ingredient 
in Tamifluß. However, much of the shikimic acid manufactured are generated by an Escherichia coli that produces shikimic acid [49], [50], [51]. Liquidambar styraciflua were found to contain shikimic acid in the bark and seeds [52], [53] and can potentially produce commercial quantities. 25-Acetoxy-3 $\alpha$-en-28-oic acid and $3 \beta$, 25-epoxy-3 $\alpha$ hydroxylup-20(29)-en-28-oic acid isolated from the cones of Liquidambar styraciflua showed moderate anti-tumor promoter [54].

In the Amaryllidaceae family, leachates from L. radiata and L. aurea all highly inhibited the lettuce radicle elongation. The Amaryllidaceae family are mostly cultivated as ornamental plants and some are used as folk medicines for the treatment of some ailments [55]. The genus Lycoris comprises about 20 species that are wildly distributed in eastern Asia wood-lands, China and Japan in particular [56]. The allelochemical in L. radiata has been identified as lycorine [57]. However, allelopathy of $L$. aurea have not been reported. The bulb of $L$. aurea have been used in China to heal fractured bones [58]. Lycosinine A \& B have been isolated from the bark of this species [59]. New alkaloids such as $2 \alpha-$ hydroxy-6-O-n-buty-loduline, O-n-butyllycorenine and (-)-N-(Chloromethyl) lycoramine have been isolated from the bulb of $L$. aurea. All the compounds exhibited significant neuro-protective effects against $\mathrm{CoCl}_{2}$ and $\mathrm{H}_{2} \mathrm{O}_{2}$-induced $\mathrm{Sh}$ SY5Y cell death [55]. Pi et al., [60] reported that some alkaloids isolated from bulb of L. aurea showed significant cytotoxicity against all tumor cell line (seven) tested. The alkaloids 3 -0-ethyltazettinol $2 \alpha$-methoxy-6-O-ethyloduline have also been isolated from the bulb of L. aurea [61], [62].

\section{Conclusion}

The results from this study hereby provide brief insight on the allelopathic potentials of some plants in the SinoJapanese Floristic Region. Further research can be conducted on the identification and characterization of allelochemicals using this data as benchmark information. Information as such could aid in the development of bioactive compounds from plant species into natural herbicides and also the utilization of these plants in sustainable weed control. We will present in our subsequent report the allelochemical(s) responsible for the inhibitory activity in Photinia glabra which was the strongest allelopathic species in this study.

\section{Acknowledgements}

This research was supported by the grant-in-aid for research work on Agriculture and Food Science (25029AB) from the Ministry of Agriculture, Forestry and Fisheries of Japan. This work was also supported by JSPS KAKENHI Grant Number 26304024.

\section{Conflict of interest}

The authors declare that there is no conflict of interest associated with this publication.

\section{References}

[1] Fujii, Y., Hiradate, S. (2007). Allelopathy: New Concepts and Methodology. Enfield, USA. Science Publishers

[2] de Albuquerque, M. B., Dos Santos, R. C., Lima, L. M., Melo- Filho, P. A., Nogueira, R. J. M. C., da C^amara, C. A. G. et al. (2011). Allelopathy, an alternative tool to improve cropping system. A review. Agronomy for Sustainable Development. 31, 379-395. http://dx.doi.org/10.1051/agro/2010031.

[3] Callaway, R. M., Ridenour, W. M. (2004). Novel Weapons: Invasive Success and the Evolution of Increased Competitive Ability. Frontiers in Ecology Environment. 2(8), 436-443. http://dx.doi.org/10.1890/1540-9295(2004)002[0436:NWISAT]2.0.CO;2.

[4] Bhadoria, P. B. S. (2011). Allelopathy: A Natural Way towards Weed Management. American Journal of Experimental Agriculture. 1(1), 7-20.

[5] Heap, I. (2014). The International Survey of Herbicide Resistant Weeds. Online. Internet. Available www.weedscience.org Accessed on Wednesday, April 22, 2015.

[6] Hall, J. C., Van Eerd, L. L., Miller, S. D., Owen, M. D. K., Prather, T. S., Shaner, D. L., Singh, M., Vaughn, K. C., Weller, S. C. (2000). Future Research Directions for Weed Science. Weed Technology 14, 647-658. http://dx.doi.org/10.1614/0890037X(2000)014[0647:FRDFWS]2.0.CO;2.

[7] Duke, S. O. (2010). Allelopathy: Current status of research and future of the discipline: A commentary. Allelopathy Journal. 2(1), 17-30.

[8] Vyvyan, J. R. (2002). Allelochemicals as leads for new herbicides and agrochemicals. Tetrahedron. 58, $1631-1646$. http://dx.doi.org/10.1016/S0040-4020(02)00052-2.

[9] Macias, F. A., Marin, D., Oliceros-Bastidas, A. et al. (2003). Allelopathy as a new strategy for sustainable ecosystems development. Biological Sciences in Space. 17(1), 18-23. http://dx.doi.org/10.2187/bss.17.18.

[10] Duke, S. O., Dayan, F. E., Romagni, J. G., Rimando, A. M. (2000). Natural products as sources of herbicides: Current status and future trends. Weed Research 40 99-111. http://dx.doi.org/10.1046/j.1365-3180.2000.00161.x.

[11] Willer, H., Lukas, K. (Eds.) (2009). The World of Organic Agriculture. Statistics and Emerging Trends 2009. FIBL-IFOAM Report. IFOAM, Bonn; FiBL, Frick; ITC, Geneva.

[12] Kropff, M. J., Walter, H. (2000). EWRS and the challenges for weed research at the start of a new millennium. Weed research 40, 7-10. http://dx.doi.org/10.1046/j.1365-3180.2000.00166.x.

[13] Wu, C. Y. (1998). Delineation and Unique Features of the Sino-Japanese Floristic Region. David E. Boufford and Hideaki Ohba (eds.), University of Tokyo Bulletin 37: Sino-Japanese Flora - Its Characteristics and Diversification (1998). Tokyo: University of Tokyo. http://www.um.u-tokyo.ac.jp/publish_db/Bulletin/no37/no37002.html. Accessed on July 15, 2015. 
[14] Qian, H. 2002. A comparison of the taxonomic richness of temperate plants in East Asia and North America. Am. J. Bot. 89, 1818-1825. http://dx.doi.org/10.3732/ajb.89.11.1818.

[15] Qian, H., R. E. Ricklefs. (2000). Large-scale processes and the Asian bias in species diversity of temperate plants. Nature 407, $180-182$. http://dx.doi.org/10.1038/35025052.

[16] Hewitt, G. M. (1996). Some genetic consequences of ice ages, and their role in divergence and speciation. Biological Sciences 58 247-276.

[17] Fujii, Y., Shibuya, T., Nakata, K., Itani, T., Hiradate, S., Parvez, M. M. (2004). Assessment Method for the Allelopathic Effect from Leaf Leachate. Weed Biology Management 4, 19-23. http://dx.doi.org/10.1111/j.1445-6664.2003.00113.x.

[18] Fujii, Y., Parvez, S. S., Parvez, M. M., Ohmae, Y., Iida, O. (2003). Screening of 239 medicinal plant species for allelopathic activity using the sandwich method. Weed Biology and Management 3, 233-241. http://dx.doi.org/10.1046/j.1444-6162.2003.00111.x.

[19] Shiraishi, S., Watanabe, I., Kuno, K., Fujii, Y. (2002). Allelopathic activity of leaching from dry leaves and exudate from roots of ground cover plants assayed on agar. Weed Biology Management 2, 133-142. http://dx.doi.org/10.1046/j.1445-6664.2002.00063.x.

[20] Morikawa, C. I. O., Miyaura, R., Segovia, G. V., Salgado, E. L. R., Fujii, Y. (2012). Evaluation of Allelopathic Activity from Peruvian plant species by sandwich method. Pak. J. Weed Sci. Res. 18, 829-834.

[21] Fujii, Y., Minoru, M., Syuntaro, H., Hideki, S. (2005). Dish pack method: A new bioassay for volatile allelopathy, Proceedings of the 4th World Congress on Allelopathy, 4: 493-497.

[22] Amini, S., Azizi, M., Joharchi, M. R., Shafei, M. N., Moradinezhad, F., Fujii, Y. (2014). Determination of allelopathic potential in some medicinal and wild plant species of Iran by dish pack method. Theoretical and Experimental Plant Physiology 26(2), 189-199. http://dx.doi.org/10.1007/s40626-014-0017-z.

[23] Gilman, E. F., Watson, D. G. (2011). Photinia glabra: Red-Photinia Environmental Horticulture, Florida Cooperative Extension Service, Institute of Food and Agricultural Sciences, University of Florida. Original publication date November 1993. Revised March 2007. Reviewed May 2011. Visit the EDIS website at http://edis.ifas.ufl.edu/st442. Assessed on June 23, 2015.

[24] Widyastuti, S. M., Nonaka, F., Watanabe, K., Sako, N., Tanaka, K. (1992). Isolation and Characterization of Two Aucuparin-Related Phytoalexins from Photinia glabra Maxim Ann. Phytopath. Soc. Japan 58, 228-233. http://dx.doi.org/10.3186/jiphytopath.58.228.

[25] Widyastuti, S. M. (2001). Sensitivity among Pestalotiopsis spp. against the Phytoalexins of Three Rosaceae Plants. Pakistan Journal of Biological Sciences 4(5), 511-513. http://dx.doi.org/10.3923/pjbs.2001.511.513.

[26] Hirai, M. (1981). Purification and Characteristics of Sorbitol-6-phosphate Dehydrogenase from Loquat Leaves. Plant Physiol. 67, $221-224$. http://dx.doi.org/10.1104/pp.67.2.221.

[27] Ishikura, N. (1975). A Survey of Anthocyanins in Fruits of Some Angiosperms. Bot. Mag. Tokyo 88, 41-45. http://dx.doi.org/10.1007/BF02498879.

[28] Marble, C. C. (2013). Birds and All Nature in Natural Colors: A Monthly Serial (Vol. 6). London: Forgotten Books. pp. 187-188 (Original work published on 1900).

[29] Shaw, L. H., Chen, W. M., Tsai, T. H. (2013). Identification of Multiple Ingredients for a Traditional Chinese Medicine Preparation (Bu-yanghuan-wu-tang) by Liquid Chromatography Coupled with Tandem Mass Spectrometry. Molecules 18, 11281-11298. http://dx.doi.org/10.3390/molecules180911281.

[30] Cheng, Z. P., Chen, Z. W., Hu, C. G., Deng, X. X. (2002). Study on Genetic Diversity of Amygdalus persica based on RAPID Markers. Journal of Wuhan Botanical Research 20(2), 89-99 (In Chinese with English abstract).

[31] Qin, S., Xue, S., Liang, Z. X., Li, G. Y., Xie, G. H. (2013) Assessment and screening of non-food biodiesel plant resources in Shaanxi and Gansu Provinces based on grey relation analysis. Journal of China Agricultural University 18(6), 6-17 (In Chinese with English abstract).

[32] Moore, M. (2001). Individual Drugs. Monographs extracted from The Eclectic Materia Medica, Pharmacology and Therapeutics by Harvey Wickes Felter, M.D. (1922).

[33] Kim, K. Y. 2005. Hibiscus. In J. L. Longe. The Gale Encyclopedia of Alternative Medicine. Farmington Hills, MI: Thomson/Gale. ISBN 0787693960 .

[34] Kawase, M., Takahashi, M. (1995). Chemical Composition of Sporopollenin in Magnolia grandiflora (Magnoliaceae) and Hibiscus syriacus (Malvaceae). Grana, 34(4), 242-245. http://dx.doi.org/10.1080/00173139509429052.

[35] Yoo, I. D., Yun, B. S., Lee, I. K., Ryoo, I. J., Choung, D. H., Han, K. H. (1998) Three Naphthalenes from root bark of Hibiscus syriacus. Phytochemistry 47(5), 799-802. http://dx.doi.org/10.1016/S0031-9422(97)00674-2.

[36] Yun, B. S., Ryoo, I. J., Lee, I. K., Yoo, I. D. (1998a). Hibispeptin A, a Novel Cyclic Peptide from Hibiscus syriacus. Tetrahedron 39, 993-996. http://dx.doi.org/10.1016/S0040-4039(97)10797-3.

[37] Yun, B. S., Ryoo, I. J., Lee. I. K., Yoo, I. D. (1998b). Hibispeptin B, a Novel Cyclic Peptide from Hibiscus syriacus. Tetrahedron 54:1515515160 http://dx.doi.org/10.1016/S0040-4020(98)00967-3.

[38] Kueffer, C., Mauremootoo, J. (2004). Case Studies on the Status of invasive Woody Plant Species in the Western Indian Ocean. 3. Mauritius (Islands of Mauritius and Rodrigues). Forest Health \& Biosecurity Working Papers FBS/4-3E. Forestry Department, Food and Agriculture Organization of the United Nations, Rome, Italy.

[39] Ganjare, A. B., Nirmal, S. A., Patil, A. N. (2011). Use of apigenin from Cordia dichotoma in the treatment of colitis. Fitoterapia 82, 10521056 http://dx.doi.org/10.1016/j.fitote.2011.06.008

[40] Pawar, H. A., Jadhav, P. (2015). Isolation, characterization and investigation of Cordia dichotoma fruit polysaccharide as an herbal excipient. International Journal of Biological Macromolecules 72, 1228-1236 http://dx.doi.org/10.1016/j.ijbiomac.2014.10.048.

[41] Singh, R, Lawania, R. D., Mishra, A., Gupta, R. (2010). Role of Cordia dichotoma seeds and leaves extract in degenerative disorders. International Journal of Pharmaceutical Sciences Review and Research 2(1), 21-24.

[42] Ahmad, I., Beg, A. Z. (2001). Antimicrobial and phytochemical studies on 45 Indian medicinal plants against multi-drug resistant human pathogens. Journal of Ethnopharmacology 74, 113-123. http://dx.doi.org/10.1016/S0378-8741(00)00335-4.

[43] Katolkar, P. P., Wanjari, B. E., Nimbekar, T. P., Duragkar, N. J. (2012). Antiimplantation activity of the methanolic extract of Cordia dichotoma Lam. Bark in rats. International Journal of Biomedical and Advance Research 3(3), 202-204.

[44] Thirupathi, K., Kumar, S. S., Raju, V. S., Ravikumar, B., Krishma, D. R., Mohan, G. K. (2008). A review of medicinal plants of the genus Cordia: Their chemistry and pharmacological uses. Journal of Natural Remedies 8(1), 1-10.

[45] Fernandez, X., Lizzani-Cuvelier, L., Loiseau, A. M., Perichet, C., Delbecque, C., Amaudo, J. F. (2005). Chemical composition of the essential oils from Turkish and Honduras Styrax. Flavour Fragr. J. 20, 70-73. http://dx.doi.org/10.1002/ffj.1370.

[46] El-Readi, M. Z., Eid, H. H., Ashour, M. L., Eid, S. Y., Labib, R. M., Sporer, F., Wink., M. (2013). Variations of the chemical composition and bioactivity of essential oils from leaves and stems of Liquidambar styraciflua (Altingiaceae). Journal of Pharmacy and Pharmacology 65, 1653-1663. 543 http://dx.doi.org/10.1111/jphp.12142.

[47] Rasmussen, R. A. (1972). What Do the Hydrocarbons from Trees Contribute to Air Pollution? Journal of the Air Pollution Control Association, 22(7), 537-543 http://dx.doi.org/10.1080/00022470.1972.10469676.

[48] Fang, C. W., Monson, R. K., Cowling, E. B. (1996). Isoprene emission, photosynthesis, and growth in sweetgum (Liquidambar styraciflua) seedlings exposed to short- and long-term drying cycles. Tree Physiology 16, 441-446. http://dx.doi.org/10.1093/treephys/16.4.441.

[49] Bradley, D. (2005). Star role for bacteria in controlling flu pandemic? Nature Reviews Drug Discovery 4, 45-46. http://dx.doi.org/10.1038/nrd1917. 
[50] Krämer, M., Bongaerts, J., Bovenberg, R., Kremer, S., Muller, U., Orf, S., Wubbolts, M., Raeven, L. (2003). Metabolic engineering for microbial production of shikimic acid. Metabolic Engineering 5, 277-283. http://dx.doi.org/10.1016/j.ymben.2003.09.001.

[51] Johansson, L., Lindskog, A., Silfversparre, G., Cimander, C., Nielsen, K. F., Liden, G. (2005). Shikimic Acid Production by a Modified Strain of E. coli (W3110.shik1) Under Phosphate-Limited and Carbon-Limited Conditions. Biotechnology and Bioengineering 92(5), 541-552 http://dx.doi.org/10.1002/bit.20546.

[52] Martin, E., Duke, J., Pelkki, M., Clausen, E. C., Carrier, D. J. (2010). Sweetgum (Liquidambar styraciflua L.): Extraction of Shikimic Acid Coupled to Dilute Acid Pretreatment. Appl. Biochem. Biotechnol. 162, 1660-1668. http://dx.doi.org/10.1007/s12010-010-8947-7.

[53] Enrich, L. B., Scheuermann, M. L., Mohadjer, A., et al (2008). Liquidambar styraciflua: a renewable source of shikimic acid. Tetrahedron Letters 49, 2513-2515. http://dx.doi.org/10.1016/j.tetlet.2008.02.140.

[54] Fukuda, Y., Sakai, K., Matsunaga, S., Tokuda, H., Tamaka, R. (2005). Cancer Chemo preventive Acidity of Lupane- and Oleanane-Type Triterpenoids from the cones of Liquidambar styraciflua. Chemistry \& Biodiversity 2, 421-428. http://dx.doi.org/10.1002/cbdv.200590021.

[55] Jin, A., Li, X., Zhu, Y. Y., Yu, H. Y., Pi, H. F., Zhang, P., Ruan, H. L. (2014). Four new compounds from the bulbs of Lycoris aurea with neuroprotective effects against $\mathrm{CoCl}_{2}$ and $\mathrm{H}_{2} \mathrm{O}_{2}$-induced $\mathrm{SH}-\mathrm{SY} 5 \mathrm{Y}$ cell injuries. Arch. Pharm. Res. 37 315-323 http://dx.doi.org/10.1007/s12272-013-0188-1.

[56] Hayashi, A., Saito, T., Mukai, Y., Kurita, S., Hori, T. A. (2005). Genetic variations in Lycoris radiata var. radiata in Japan. Genes Genet. Syst. 80, 199-212. http://dx.doi.org/10.1266/ggs.80.199.

[57] Iqbal, Z., Zasir, H., Hiradate, S., Fujii, Y. (2006). Plant growth inhibitory activity of Lycoris radiata Herb. and the possible involvement of lycorine as an allelochemical. Weed Biology and Management 6, 221-227. http://dx.doi.org/10.1111/j.1445-6664.2006.00217.x.

[58] Yang, Y., Huang, S. X., Zhao, Y. M., Zhao, Q. S., Sun, H. D. (2005). Alkaloids from the Bulbs of Lycoris radiata. Helvetica Chimica Acta 88, 2550-2553. http://dx.doi.org/10.1002/hlca.200590193.

[59] Lee, S., Xiao, C., Pei, S. (2008). Ethnobotanical survey of medicinal plants at periodic markets of Honghe Prefecture in Yunnan Province, SW China. Journal of Ethnopharmacology 117, 362-377. http://dx.doi.org/10.1016/j.jep.2008.02.001.

[60] Song, J. H., Zhang, L., Song, Y. (2014). Alkaloids from Lycoris aurea and their cytotoxicities against the head and neck squamous cell carcinoma. Fitoterapia 95, 121-126. http://dx.doi.org/10.1016/j.fitote.2014.03.006.

[61] Pi, H. F., Zhang, P., Ruan, H. L., Zhang, Y. H., Sun, H. D., Wu, J. Z. (2009). A new alkaloid from Lycoris aurea. Chinese Chemical Letters 20, 1319-1320. http://dx.doi.org/10.1016/j.cclet.2009.06.003.

[62] Huang, S. D., Zhang, Y., He, H. P. et al., (2013). A new Amaryllidaceae alkaloid from the bulbs of Lycoris radiata. Chinese Journal of Natural Medicine 11(4), 406-410. http://dx.doi.org/10.1016/S1875-5364(13)60060-6. 